\title{
Dinámica geomorfológica y medio ambiente en los sitios arqueológicos Chirije y San Jacinto/Japoto (costa del Manabí central, Ecuador)
}

Dynamique géomorphologique et environnement des sites archéologiques de Chirije et Japoto/San Jacinto (côte du Manabí central, Équateur)

Dynamic geomorphologic and environment in the archaeological sites Chirije and San Jacinto/Japoto (the central coast of Manabi, Ecuador)

\section{Pierre Usselmann}

\section{OpenEdition}

Journals

Edición electrónica

URL: http://journals.openedition.org/bifea/3624

DOI: $10.4000 /$ bifea.3624

ISSN: 2076-5827

Editor

Institut Français d'Études Andines

Edición impresa

Fecha de publicación: 1 diciembre 2006

Paginación: 257-264

ISSN: 0303-7495

Referencia electrónica

Pierre Usselmann, « Dinámica geomorfológica y medio ambiente en los sitios arqueológicos Chirije y San Jacinto/Japoto (costa del Manabí central, Ecuador) », Bulletin de l'Institut français d'études andines [En línea], 35 (3) | 2006, Publicado el 01 junio 2007, consultado el 02 diciembre 2020. URL : http:// journals.openedition.org/bifea/3624 ; DOI : https://doi.org/10.4000/bifea.3624

\section{cc)}

Les contenus du Bulletin de l'Institut français d'études andines sont mis à disposition selon les termes de la licence Creative Commons Attribution - Pas d'Utilisation Commerciale - Pas de Modification 4.0 International. 


\title{
Dinámica geomorfológica y medio ambiente en los sitios arqueológicos Chirije y San Jacinto/Japoto (costa del Manabí central, Ecuador)
}

\author{
Pierre Usselmann*
}

\section{Resumen}

La costa del Manabí central presenta la alternancia de acantilados rocosos y de llanuras litorales de relleno con lagunas. Los sitios arqueológicos estudiados pertenecen a los dos ambientes que ofrecen una gran biodiversidad gracias a la riqueza marina, a los pequeños valles de los esteros y a las lagunas de manglares. La desaparición de este manglar ha tenido seguramente un papel importante en la desestabilización de los grandes establecimientos humanos explotadores de este medio ambiente.

Palabras clave: Ecuador, Manabí central, Chirije, Japoto/San Jacinto, dinámica geomorfológica, medio ambiente

\section{Dynamique géomorphologique et environnement des sites archéologiques de Chirije et Japoto/San Jacinto (côte du Manabí central, Equateur)}

\section{Résumé}

La côte du Manabí central présente une succession de falaises rocheuses et de plaines littorales de remblaiement à lagunes. Les sites archéologiques étudiés concernent les deux milieux qui offraient une grande biodiversité grâce à la richesse marine, à celle des petites vallées des esteros et des 
lagunes à mangrove. La disparition de cette mangrove a vraisemblablement joué un grand rôle dans la déstabilisation des grands établissements humains qui I'exploitaient.

Mots clés : Équateur, Manabí central, Chirije, Japoto/San Jacinto, dynamique géomorphologique, environnement

\title{
Dynamic geomorphologic and environment in the archaeological sites Chirije and San Jacinto/Japoto (the central coast of Manabi, Ecuador)
}

\begin{abstract}
The central coast of Manabi presents an alternation of rocky cliffs and coastal fill plains with ponds. The studied archaeological places belongs to both environments and presents a great biological diversity due to its richness sealife, the small marsh valleys and the swamp ponds of the mangrove. Probably the dissapearence of this mangrove have played an important role on the unbalance of the big human settlements exploiters of this enviroment.
\end{abstract}

Key words: Ecuador, central Manabi, Chirije, Japoto/San Jacinto, dynamic geomorphologic, environment

La costa del Manabí central —ubicada debajo de la línea equinoccial, inmediatamente al sur de Bahía de Caraquéz - se caracteriza por un clima seco y caliente. Se observa un promedio de 10 hasta 11 meses secos al año (Winckell \& Zebrowski, 1997) con una temperatura media de $25^{\circ}$. Las precipitaciones alcanzan normalmente 300 hasta $600 \mathrm{~mm}$ al año, pero estas cantidades pueden ser ampliamente superadas durante los episodios ENSO (EI Niño Southern Oscillation).

La vegetación es xerofítica en las bajas alturas, está compuesta de matorrales con arbustos espinosos (monte espinoso tropical) donde sobresalen las altas ceibas. Sin embargo, sobre los cien metros de altura, la presencia de nieblas costaneras a lo largo del año favorece el desarrollo de bosques nítidamente más húmedos (Estrada, 1962). Al igual que en los Andes, el escalonamiento altitudinal se traduce entonces por cambios rápidos, cuyos efectos han sido siempre importantes para las poblaciones.

Los suelos, debido a la sequía, están poco evolucionados, solo cuentan con un horizonte superficial más o menos humífero. Se desarrollan sobre rocas poco resistentes, lutitas y hasta areniscas blandas que suministran por meteorización sedimentos de textura areno-limosa con un cierto contenido en arcillas y un gran poder expansivo (tipo montmorillonitas).

Al pie de las bajas colinas (350 $\mathrm{m}$ de altura en promedio), el litoral propiamente dicho se presenta de manera contrastada a lo largo de unos treinta kilometros al sur de Bahía de Caraquéz: abruptos acantilados ocupan la parte norte mientras que, hacia el sur, se desarrolla una llanura costera de cordones litorales y ciénagas. Una corriente marina costera, con un flujo sur-norte, confiere a la costa este aspecto rectilíneo muy aparente en el mapa topográfico. Los sitios arqueológicos estudiados en el marco del Programa Manabí se encuentran en estos dos conjuntos. 


\section{EL PAISAJE DE LOS ACANTILADOS}

Entre Bahía de Caraquéz y San Clemente los acantilados alcanzan unos cincuenta metros de altura, recortados perpendicularmente por varios pequeños valles estrechos; estos últimos están esporádicamente o estacionalmente barridos por las aguas de torrentes. Los esteros permanecen sin agua la mayor parte del tiempo. Cortados en rocas blandas recientes (Terciario) de lutitas, margas con yeso, calizas y areniscas, los acantilados se encuentran continuamente atacados en sus bases por el océano, y la costa presenta el aspecto típico de una costa de erosión.

Al pie de los acantilados se encuentra, en marea baja, una playa de un centenar de metros de ancho. Los eventos ENSO (o El Niño, Arteaga et al., 2006; Cobo, 2001) —cuya última fuerte manifestación en 1997-1998 ha dejado huellas aún importantes-suministran agua y una gran cantidad de material a los esteros; al mismo tiempo, los acantilados y las laderas de los pequeños valles presentan importantes y variados movimientos en masa (Santana et al., 2001, Plaza et al., 2000). La llegada de todos estos sedimentos a la costa provoca acumulaciones superiores a las posibilidades inmediatas de movilización del océano y de su corriente costera. Un nivel superior de playa se desarrolla entonces rápidamente sobre unos $2 \mathrm{~m}$ de altura al pie de los acantilados, protegiéndoles de la erosión marina directa durante cierto tiempo. Este depósito se ve poco a poco arrastrado por las olas y los

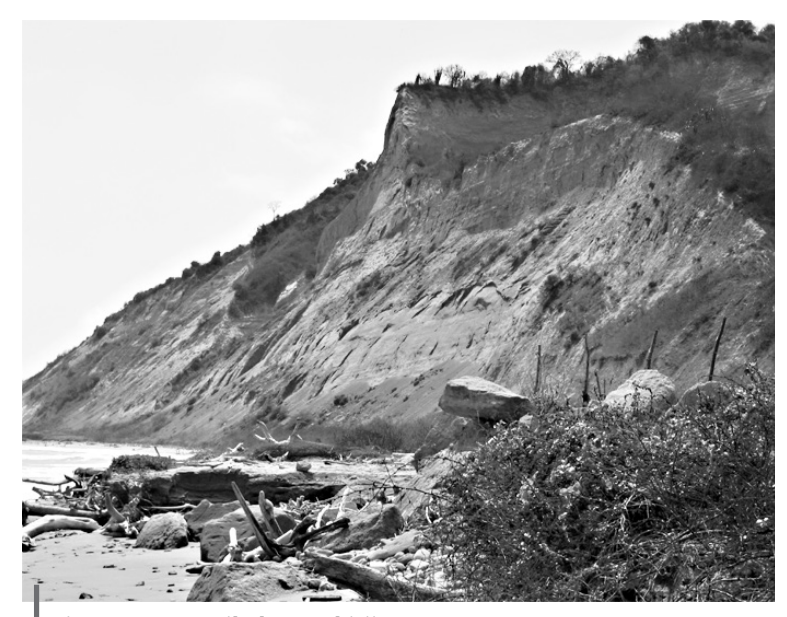

Figura 1 - Acantilado en Chirije

En primer plano se puede apreciar lo que queda del nivel superior de playa ligado al Niño 1997-1998 sedimentos son transportados entonces hacia el norte (fig. 1). Esta dinámica global se encuentra ilustrada en la cerradura de estos sedimentos del estuario del río Chone en Bahía de Caraquéz (Tutiven, 1998), lo que se produce también en el sur, en el estuario del río Portoviejo.

El sitio arqueológico de Chirije (fig. 2) se ubica sobre los abanicos-terrazas de los esteros Chirije Grande y Chirije Chico encajonados en las colinas. Se estableció — prácticamente— en el lugar donde estas colinas, cortadas por el océano, forman altos acantilados y se presenta bien caracterizado dentro de todos estos valles cuyas acumulaciones aluviales se encuentran troncadas por el océano.

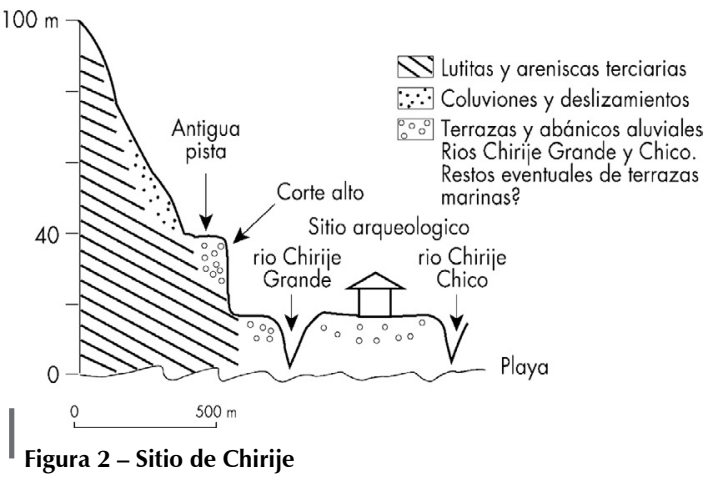




\section{LA COSTA BAJA}

Se trata de una costa fluvio-lagunar de acumulación —el río Portoviejo desemboca en el océano inmediatamente al sur de San Jacinto- de unos 3 kilómetros de ancho. Se observan, desde el océano y hacia el interior (fig. 3): un cordón litoral arenoso composito (un cordón antiguo de unos 200 a 300 metros de ancho, un cordón actual muy reducido); algunas lagunas actuales o antiguas ciénagas rellenadas; en fin, un glacis de acumulación entre las lagunas y las bajas colinas cercanas, donde se ubica el sitio arqueológico de Japoto/San Jacinto. Este nivel ha sido constituido por los aportes sedimentorios de la escorrentía superficial y de los esteros. Estos pequeños torrentes llegan a las ciénagas (caso del sitio de Japoto) o directamente al río Portoviejo.

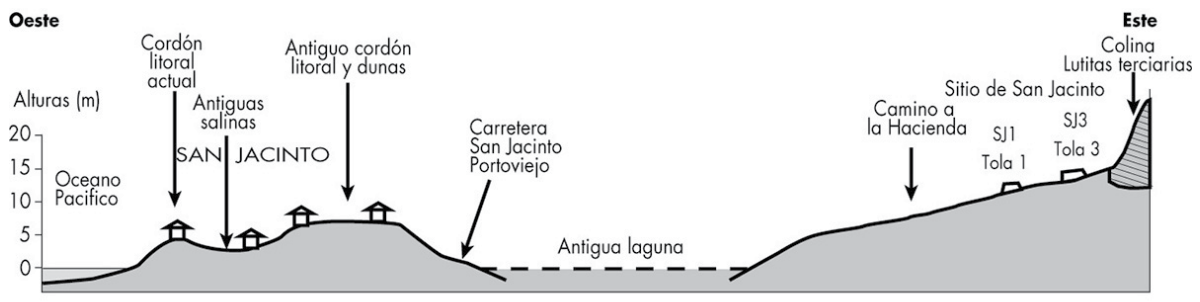

\footnotetext{
Figura 3 - Perfil topográfico oeste-este a la altura de San Jacinto
}

Como en el caso de los acantilados, las laderas de las bajas colinas están siempre constituidas por rocas en general blandas, aptas para el suministro de grandes cantidades de sedimentos - particularmente en el caso de los eventos ENSO - pero también durante las estaciónes Iluviosas habituales. Sin embargo, las lutitas y areniscas blandas contienen niveles más consolidados y concretos aptos para suministro de rocas duras aprovechadas por los antiguos pobladores — particularmente para pulir sus cerámicas-.

Como en el pasado, los fenómenos de relleno dominan en esta parte baja: se encuentran favorecidos por la naturaleza de los suelos y de las rocas de las laderas (fig. 4), las que favorecen a su vez a los aportes finos de los esteros y de las laderas locales (fig. 5). También por el hecho de que la mayor parte de los caudales actuales del río Portoviejo están consumidos por los importantes riegos a lo largo de todo el valle. De este modo, la desembocadura del río se encuentra con mucha menos agua que la prevista. Esta dinámica ha favorecido el relleno de la gran laguna de San Clemente/San Jacinto, su aislamiento por la ausencia de comunicación con el océano y, en consecuencia, la desaparición de un manglar litoral cuyos pocos restos son todavía visibles en los márgenes del río Portoviejo en su desembocadura.

\section{UNA DINÁMICA ACTIVA MARCADA TAMBIÉN POR INFLUENCIAS EXTERNAS}

La cartografía esquemática del sitio de Japoto (fig. 6) muestra la coexistencia de pequeños conjuntos con dominio de sedimentos coluviales (escorrentía superficial) o de sedimentos de inundaciones o deslaves movilizados por los esteros. Estos sedimentos son finos en general, pero pueden contener pequeños cantos, y se juntan, aguas abajo, con sedimentos areno-limosos de las lagunas (fig. 7). Los sedimentos finos de inundación aparecen bajo la forma de una sucesion de estratos finos subhorizontales, más o menos limosos o arenosos, en los cuales se encuentran evidentemene restos antrópicos, particularmente tiestos de cerámica. 
Dinámica geomorfológica y medio ambiente en los sitios arqueológicos Chirije y San Jacinto/Japoto

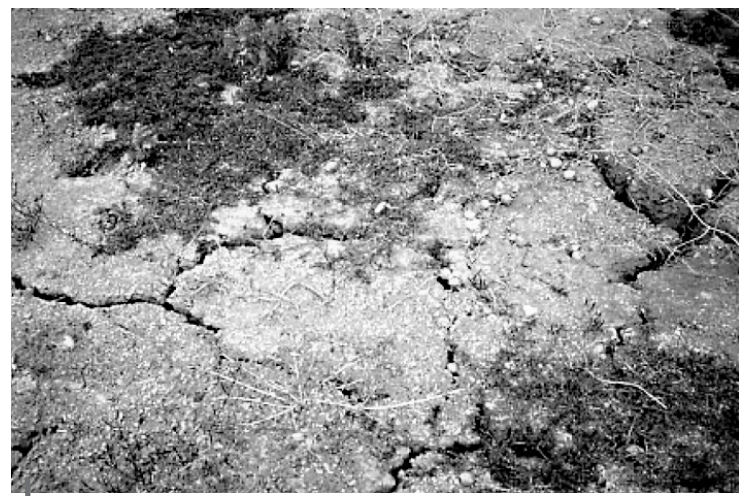

Figura 4 - Grietas de sequía en formaciones superficiales de laderas cerca de Japoto/San Jacinto

Estas grietas favorecen la penetración del agua en época de lluvias

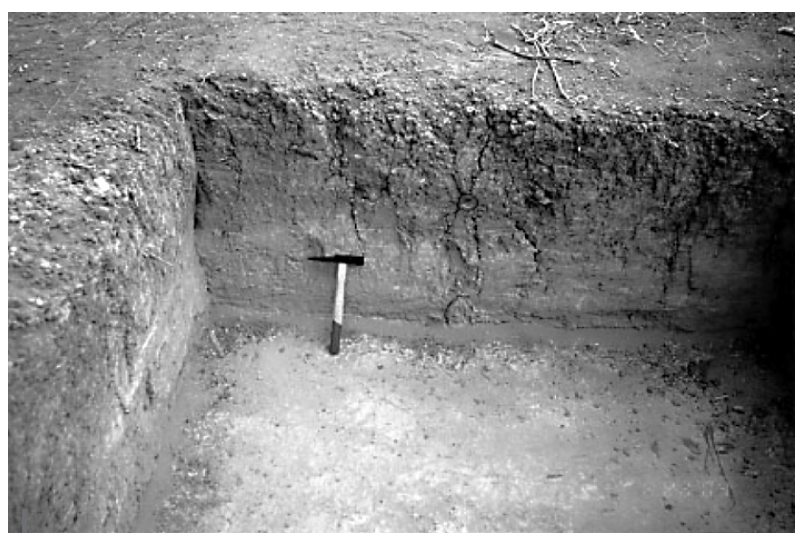

Figura 5 - Sedimentos finos coluvio-aluviales en el fondo del cauce de un estero

Se observa una sucesión de delgados estratos subhorizontales

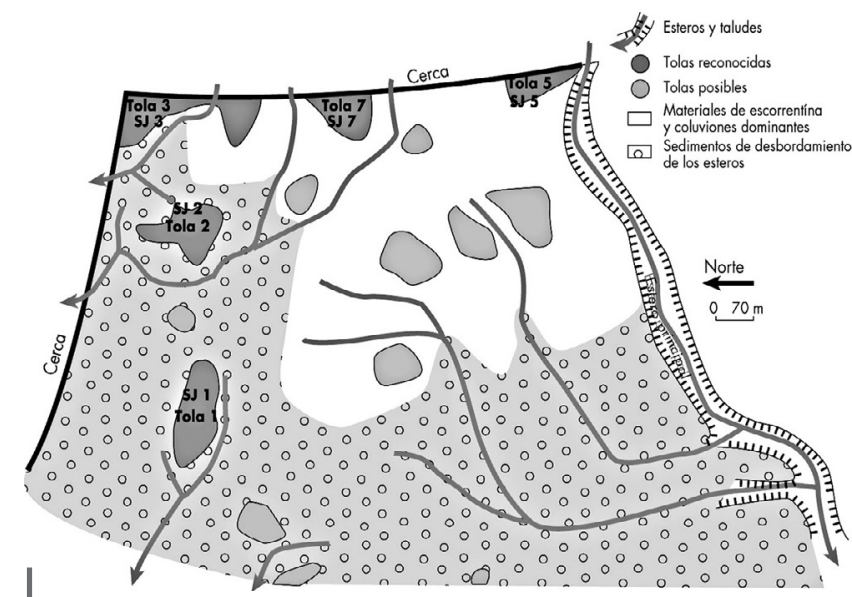

Figura 6 - Esquema del entorno físico de Japoto/San Jacinto 


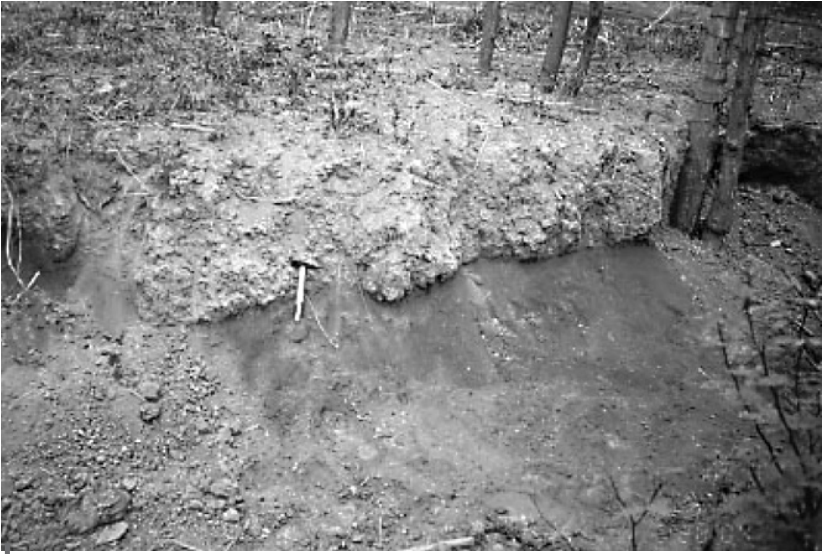

Figura 7 - Acumulaciones de inundaciones de los esteros (arriba) fosilizando sedimentos areno-limosos lagunares, en la parte baja del sitio Japoto/San Jacinto (oeste)

Pero lo que hay que subrayar es la omnipresencia de cenizas volcánicas (llamadas a menudo y abusivamente tefras 1 ) en el paisaje y en los sedimentos. En el Ecuador, numerosos volcanes siguen actualmente activos o lo fueron recientemente $y$ en el pasado; sus erupciones han brotado grandes cantidades de cenizas, dispersas a los dos lados de la Cordillera y del valle central del Ecuador. En Chirije como en Japoto, estas cenizas son siempre removidas y mezcladas con limos y arcillas. Su movilización se hace y se ha hecho por la escorrentía superficial y por los flujos de los esteros. Se encuentran restos y testigos en las laderas (fig. 8) donde pueden moldear antiguas topografías. Desafortunadamente, no disponemos hasta la fecha de dataciones absolutas de estas cenizas. No muy lejos, al norte de la región de estudio, en los alrededores de Jama, Zeidler \& Pearsall (1994) atribuyen cenizas del mismo tipo a unas erupciones de los volcanes Atacazo/Ninahuilca (más probable) o Tungurahua fechadas de 300 a 500 años de nuestra era (posteriores al Jama Coaque mediano). Mothes (1998) y Hall \& Mothes (2006) hablan de una gran actividad volcánica más reciente entre 1000 y 800 años BP (alrededor de $1000 \mathrm{AD}$ ) que afectó a gran parte del Ecuador, particularmente a partir del Quilotoa y eventualmente del Cotopaxi y del Pululahua. Sin embargo, no sabemos si las cenizas de Japoto pueden pertenecer a estos acontecimientos. La dirección dominante del este hacia el oeste de los vientos por encima de los Andes ecuatorianos explicaría la importancia de esta aspersión. Las cenizas removidas se observan en las laderas tanto sobre como dentro de los depósitos de abanicos-terrazas cercanos a los esteros; en el caso de Chirije, éstos dominan la playa actual de unos 10 metros y soportan al sitio arqueológico. Se encuentran cenizas en varios niveles en los cortes arqueológicos, como en los depósitos más antiguos de los esteros (fig. 9). En todos los casos, estas cenizas parecen, hasta ahora y a partir de los conocimientos arqueológicos actuales, constituir la referencia más antigua para los sitios de Japoto y de Chirije y han sido usadas en la construcción de las tolas para establecer suelos o revestimientos particulares.

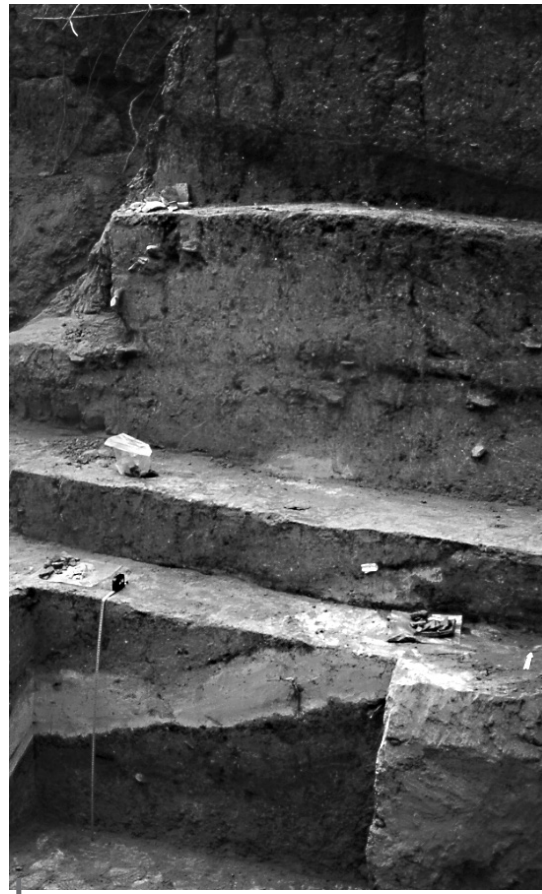

Figura 8 - Chirije, ladera norte (corte alto) Las cenizas rellenan un antiguo barranco

1 Una tefra corresponde a todo tipo de material sólido, cualquiera que sea su tamaño, fuera de una lava, emitida en el aire o bajo el agua durante erupciones volcánicas explosivas. Es la resultante de una fragmentación del magma y constituye progresivamente los conos volcánicos. 


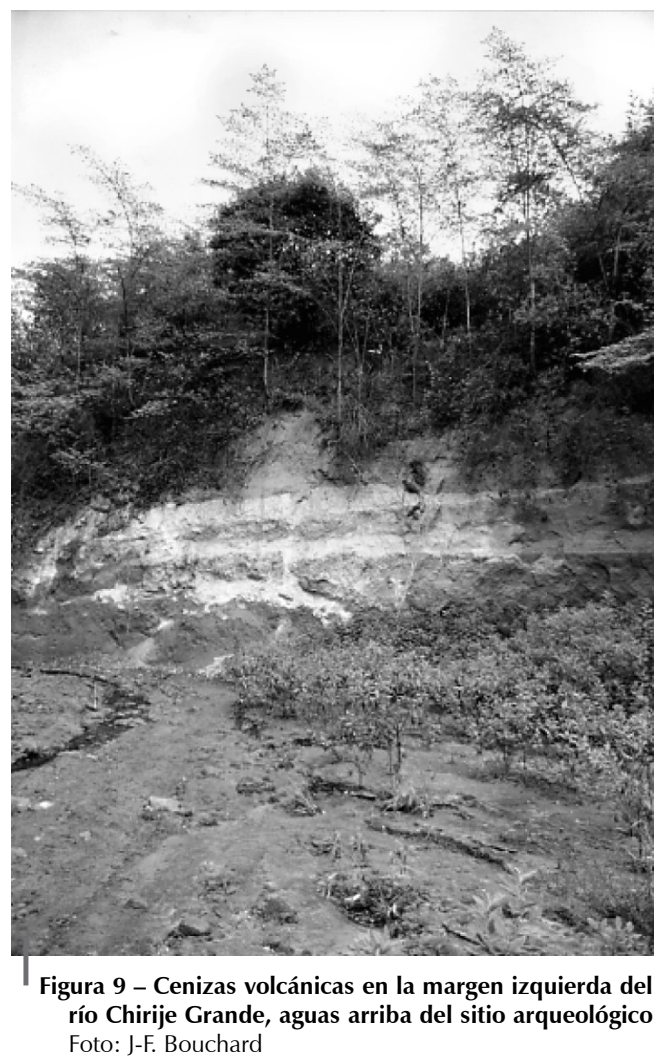

\section{CONCLUSIÓN}

El océano Pacífico sigue actualmente erosionando toda esta costa y regularizando su perfil a lo largo de una línea sur-norte: las puntas rocosas desaparecen (caso de Chirije) y el cordón litoral reciente se encuentra bastante desestabilizado, lo que pone en peligro varias construcciones de los pueblos de San Jacinto y de San Clemente. Una protección únicamente temporal e ilusoria a corta escala de tiempo se hace con el amontonamiento de rocas cercanas a las habitaciones, a lo largo de la línea costera. Los sedimentos movilizados se van desplazando hacia el norte, rellenando y taponando la desembocadura del río Chone entre Bahía de Caraquéz y San Vicente. El sitio arqueológico de Chirije, establecido sobre los abanicos terrazas de los esteros Chirije Grande y Chirije Chico, caracteriza bien el caso de dos pequeños valles cuyas acumulaciones aluviales se encuentran troncadas por el océano. En Japoto, la evolución es más completa y compleja; las principales etapas pueden reconstituirse esquemáticamente de la siguente manera, las que serán convenientemente relacionadas con la o las ocupaciones humanas: desarrollo de una costa con un importante cordón litoral debido a los aportes del río Portoviejo y a la existencia de la corriente oceánica costera sur-norte. Este cordón litoral aísla poco a poco una laguna de una extensión de varias decenas de hectareas.

Estas dos primeras etapas son antiguas, esencialmente anteriores a la ocupación antrópica.

La laguna, alimentada por las aguas dulces del río Portoviejo y comunicada también con el océano, tiene sus márgenes ocupados por un manglar; es decir, un medio natural biológicamente muy rico y apto para favorecer, en sus cercanías, el desarrollo de establecimientos humanos.

La laguna se va rellenando después por varias razones por el momento no bien establecidas: un cambio en la dirección del curso inferior del río Portoviejo ha podido producirse, lo que habría bloqueado el aporte de agua dulce a la laguna. Una crecida importante del río, por ejemplo durante un acontecimiento ENSO, podría ser responsable de un cambio de este tipo. Pero una tendencia más importante es la sequía del clima regional que podría también explicar al fenómeno, con aportes sedimentarios importantes a partir de las laderas y por los esteros durante los cortos pero intensos eventos lluviosos. En todos los casos, la disminución de la llegada de agua dulce explicaría la desaparición del manglar y, entonces, las condiciones de vida más difíciles para los habitantes. De manera opuesta, un aislamiento total del océano puede también haber tenido como consecuencia la desaparición del agua salobre necesaria para un buen desarrollo del manglar. 
Por fin, se observa actualmente un retroceso nítido del conjunto de la costa, tratándose de los acantilados o del cordón litoral, el cual está ahora protejido con enrocamientos poco eficientes. Un nuevo factor entra también en juego en este último siglo con las irrigaciones del fondo del valle del río Portoviejo, que limitan de manera drástica los caudales del río en su desembocadura. Esta reducción de los volúmenes de agua se une a una disminución de los sedimentos traídos por el río, mientras que el océano sigue moldeando la costa con la misma energía. La comparación de las fotografías aéreas de 1970 y 1994 deja ver muy claramente este retroceso.

La obtención de dataciones absolutas a partir de los carbones abundantes en las excavaciones y la cronología relativa de las cerámicas van a permitir mejorar nítidamente estas primeras propuestas.

\section{Referencias citadas}

ARTEAGA, K.,TUTASI, P. \& JIMENEZ, R., 2006 - Climatic variability related to El Niño in Ecuador. A historical background. Advances in Geosciences, 6: 237-241; Alemania: European Geosciences Union (EGU).

COBO ARIZAGA, C., 2001 - Taking advantage of El Niño climatic phenomenon in humid areas and how to control sediments of floods by reforesting Guadua massively. In: Coll. Bamboo in Desaster Advoidance (Yipin, L., ed.): 12-23; Guayaquil: INBAR (Intern. Network for Bamboo and Rattan).

ESTRADA, E., 1962 - Arqueología de Manabí Central, 210 p.; Guayaquil: Ed. Museo V.E. Estrada.

HALL, M. \& MOTHES, P., 2006 - Volcanic impediments to the development of pre-columbian cultures in the andean highlands of Ecuador; Quito. Resumen in Cities on volcanoes 4: http://www.citiesonvolcanoes4.com

MOTHES, P., 1998 - Actividad volcánica y pueblos precolombinos en el Ecuador, 205 p; Quito: Abya Yala.

PLAZA, G., GALARRAGA, R., VALVERDE, J., PROANO, O. \& JIMENEZ, E., 2000 - Zonificación del peligro por deslizamientos, flujos de lodos y escombros y sismos. El caso de la ciudad de Bahía de Caraquéz, Ecuador. In: Segundo Encuentro Académico Industrial, Jornadas de Investigación: 610-611; Caracas: Facultad de Ingenieria, Universidad Central de Venezuela. Resumen.

SANTANA, E., DUMONT, J.-F. \& KING., A., 2001 - Los efectos del fenómeno El Niño en la ocurrencia de una alta tasa de erosión costera en el sector de Punta Gorda, Esmeraldas. Acta Oceanográfica del Pacífico: 1-8; Guayaquil: INOCAR.

TUTIVEN UBILLA, I., 1998 - Variaciones morfológicas y batimétricas de la línea de costa en el estuario del Río Chone, producidas por los eventos ENSO. Bulletin de l'Institut Français d'Études Andines, 27 (3): 557-563; Lima.

WINCKELL, A. \& ZEBROWSKI, CL., 1997 - Los paisajes naturales del Ecuador, 417 p.; Quito: Instituto Geografico Militar.

ZEIDLER, J. A. \& PEARSALL, D. M., 1994 - Regional Archaeology in Northern Manabí, Ecuador. Memoirs in Latin American Archaeology, vol. 1, 224 p.; Pittsburgh/Quito: University of Pittsburgh. 